\title{
Solving Optimal Control Problem Using Max-Min Ant System
}

\author{
S. Babaeizadeh, A. Banitalebi, Rohanin Ahmad, Mohd Ismail Abd Aziz \\ Department of Mathematical Sciences, Faculty of Science, \\ Universiti Teknologi Malaysia, 81310 Skudai, Johor, Malaysia
}

\begin{abstract}
An improved ant colony algorithm for optimal control problems with box constrain on control functions is presented. The hypercube of the feasible controls as well as the time interval are initially discretized to approximate control problem into a discrete parameter selection problem. Then, the ant colony algorithm is applied to search for optimum parameters of approximated problem while a proper local search is also introduced to iteratively enhance the quality of solution. The results of numerical simulation on MATLAB environment illustrate the effectiveness of this method.
\end{abstract}

Keywords - Ant colony, Global optimization, Optimal control problem.

\section{INTRODUCTION}

Optimal control problems are generally very challenging to solve, however this form of problems arise in many real world problems in a variety of disciplines. In this study, we consider a class of control problems where the system of dynamics is governed by an ordinary differential equation, and the objective is to minimize a real valued performance index while the control functions are constrained by a hypercube as follow,

$$
\begin{aligned}
\min & j\left(\mathrm{x}\left(\mathrm{t}_{\mathrm{f}}\right)\right) \\
& x(t)=f(x(t), u(t), t) \\
& x\left(t_{0}\right)=x_{0} \\
& t_{0} £ t £ t_{f}, \quad \mathrm{x} \hat{I} \hat{\mathrm{A}}^{\mathrm{n}}, \\
& \mathrm{u} \hat{I}\left[\mathrm{a}_{1}, \mathrm{~b}_{1}\right]^{\prime} \mathrm{K}^{\prime}\left[\mathrm{a}_{\mathrm{m}}, \mathrm{b}_{\mathrm{m}}\right] \mathrm{I}^{\mathrm{I}} \hat{\mathrm{A}}^{\mathrm{m}}
\end{aligned}
$$

As it is well known, the analytical solution for above problem is not always available. Therefore, numerous computational methods are proposed to approximate a solution for this problem. In the last decade, some metaheuristics including Ant Colony Optimization (ACO) methods have also been applied to furnish some control problems with an acceptable solution when the gradient information is not available.

A number of researchers focused on developing ACO algorithms for optimal control problems. The first work on linking ACO to the control problems was proposed by Birattari, Caro, and Dorigo [1] where an iterated Monte Carlo approach was used on combinatorial optimal control problems. Then, the control problem of the pump operations in water distribution networks was solved using ACO [2]. An ant colony optimization was also proposed by Borzabadi, and Mehne [3] to solve a class of optimal control problems with single input control. Rishiwal, Yadav, and Arya [4] applied ant colony optimization for finding optimal paths on terrain map images. A novel framework called Ant Colony Learning (ACL) proposed for finding optimal control policies. In this method ants work with each other to learn optimal control policies collectively [5].

In this work we investigate to improve an efficient ACO method known as Max- Min Ant System (MMAS) by introducing a local search as a daemon action to enhance this algorithm further for the solution of control problems. In the next section ACO methods are briefly reviewed. Then, in subsequent sections Problem (1) is transformed into a combinatorial optimization problem, and solved using an improved MMAS method.

\section{ANT COLONY OPTIMIZATION}

ACO is an actively developing nature-inspired metaheuristics. The inspiring concept is based on the observation of foraging behavior of real ants which enables ants to find shortest path between their nest and food sources. This technique has been formalized by Dorigo [6], as a treatment for combinatorial optimization problem (COP). Henceforth, the popularity of ACO steadily grows, and the area of applications constantly develops.

Ant system (AS) was the first ant colony algorithm formulated by Dorigo, Maniezzo, and Colorni [7]. The main characteristic of this algorithm is that the pheromone values are updated by all the ants that have made a tour. There are several improvements on AS. The first improvement of the initial AS, called the elitist strategy for ant system (EAS) [6]. The characteristic of this method is to provide strong additional reinforcement to the 
best tour found since the start of algorithm (best-so-far tour). Rank based $\operatorname{AS}\left(A S_{\text {rank }}\right)$ is another enhancement over AS [8]. In this method pheromones are updated according to the rank of ants.

Ant Colony System (ACS) suggested by Dorigo, and Gambardella [8] is another variant of AS. The most interesting aspect of this algorithm consists of using two pheromone update rules, local and global. The pheromones are updated locally during solutions construction and a global pheromone update occurs at the end of the construction process.

MAX-MIN Ant System (MMAS) proposed by Stützle and Hoos [10], [11]. This method is a version of AS trying to effectively exploit good solutions. It can be applied to identify potential solutions in the search space using pheromone trail and create new promising initial solution for local search [10]. This advantage has inspired us to start a further investigation on applying MMAS for solving optimal control problem.

\section{DISCRETIZATION}

Problem (1) is in the continuous form while the MMAS is a combinatorial optimization method. Therefore, to apply this method on Problem (1) we use control parameterization technique to discretize this problem into a parameter selection problem. The control function then is in the form of

$$
u(t)=\stackrel{\varliminf}{a}_{i=1}^{n} u_{i} c_{\left[t_{i}, t_{i+1}\right]},
$$

where $t_{0}=t_{1} £ \mathrm{~K} £ t_{n}=t_{f}$, and the parameters $u_{i}$ are also selected from a finite set $D_{1}{ }^{\prime} \mathrm{K}^{\prime} D_{m}$ of hypercube

$$
\widetilde{\mathbf{O}}_{i=1}^{m}\left[a_{i}, b_{i}\right] \text { Í } \hat{\mathrm{A}}^{m}
$$

in Problem (1), where $D_{i}$ Í $\left[a_{i}, b_{i}\right]$. The subset $D_{i}$ can be obtained through uniformly discretizing the interval $\left[a_{i}, b_{i}\right]$.

\section{THE MAX Min ANT SYSTEM}

The key feature of MMAS is that, the pheromone trails are updated with only one ant, this ant could be the one producing the best solution in the current iteration (iteration-best ant) or the one which found the best solution from the start of the algorithm (global-best ant). Moreover, the maximum and minimum values of the pheromones are limited to certain values to escape getting stuck at local solutions. Additionally, pheromone trails initialize to upper bound $t_{\max }$ to have uniform exploration in the whole search space [6]. This algorithm is illustrated below and subsequently described further,

\section{Max-Min Ant System Algorithm}

Initialize pheromone trails, Set parameters.

While termination conditions not met do

Solution construction

Daemon Actions (Local search)

end while

Pheromone update

\subsection{Solution construction}

Artificial ants construct promising solutions componentwise. The construction mechanism start with an initially empty solution $s_{p}=Æ$, then at each construction step, a partial solution is extended by adding a feasible solution component to the current partial solution according to the probability,

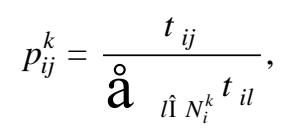

Where $t_{i j}$ is the associated pheromone trial, and $N_{i}^{k}$ is the feasible neighborhood of ant $k$ when being at position $i$.

\subsection{Daemon actions}

The available literature shows that the promising approach to refine the solution quality generated by metaheuristic methods is to incorporate a local search mechanism within the metaheuristic framework [6].

$$
\text { www.iosrjournals.org }
$$


Therefore, in order to provide higher quality solutions, while using MMAS method to solve Problem (1), local search algorithm operates on the best solution found after the solution construction and evaluation process. In this work, we use the Gaussian local search operator which is based on Gaussian distribution also known as normal distribution and often denoted by,

$$
n\left(m, s^{2}\right)=\frac{1}{\sqrt{2 p s^{2}}} e\left(-\frac{(x-m)^{2}}{2 s^{2}}\right) .
$$

This local search algorithm is applied within the MMAS algorithm as a daemon action and results in considerable improvements in the quality of solution. It is described below.

\section{Gaussian local search operator}

Initialize parameter $\min n\left(m, s^{2}\right)$ with the best solution found, and set $0<s<1$.

Generate a few trial solutions according to distribution $n\left(m, s^{2}\right)$.

Evaluate trial solutions and output the best one.

\subsection{Pheromone update}

The last operator in the MMAS algorithm updates the pheromone table in two stages; first, evaporation is applied uniformly as follow,

$$
t_{i j}=(1-r) t_{i j}
$$

where, $0<r £ 1$, and then the elements of the pheromone table associated with the best solution are increased by,

$$
t_{i j}=t_{i j}+\frac{1}{j_{\text {best }}},
$$

where $j_{\text {best }}$ is the value of cost functional according to the best solution. In addition, as mentioned above, all the pheromones are limited between $t_{\min }$ and $t_{\max }$. The value of $t_{\max }$ is computed analytically [11] by,

$$
t_{\max }=\frac{1}{(1-r)} \frac{1}{j_{\text {best }}},
$$

while the lower pheromone is computed by

$$
t_{\text {min }}=\frac{t_{\max }}{a}
$$

where $a$ is parameter [6]. Therefore, the pheromone update algorithm is as follow,

\section{Pheromone update operator}

Evaporate pheromone trials by (2).

Increase the pheromones associated with best solution by (3).

If pheromone trial $t_{i j}<t_{\text {min }}$ then set $t_{i j}=t_{\text {min }}$.

If pheromone trial $t_{i j}>t_{\text {max }}$ then set $t_{i j}=t_{\text {max }}$.

If the current best solution has not improved for a certain number of iterations reinitialize the pheromone table by setting $t_{i j}=t_{\text {max }}$ for all $i$ and $j$. 


\section{NUMERICAL RESULT}

The improved MMAS algorithm described above is evaluated using a continuous stirred tank reactor problem taken from [12],

$$
\begin{aligned}
& \min \mathrm{J}=\mathrm{x}_{3}\left(\mathrm{t}_{\mathrm{f}}\right) \\
& \&=\left(x_{2}+0.5\right) \exp \left(\frac{25 x_{1}}{x_{1}+2}\right)-(2+u)\left(x_{1}+0.25\right) \\
& \&=0.5-x_{2}+\left(x_{2}+0.5\right) \exp \left(\frac{25 x_{1}}{x_{1}+2}\right) \\
& \&=x_{1}^{2}+x_{2}^{2}+0.1 u^{2} \\
& 0 £ t £ 0.78 \quad 0 £ u(t) £ 5 \\
& x\left(t_{0}\right)=[0.09,0.09,0]^{T}
\end{aligned}
$$

This problem first was discretized using 10 and 50 discrete points in time and control intervals. Then, the ode 45 function available in MATLAB, used for the integration of the system of dynamics while the tolerance error set to $10^{-8}$. The parameter $s$ in Gaussian local search operator set to 0.7 and the number of sampling in both MMAS and local search algorithm selected to 100 samples. The evaporation parameter chosen to be 0.8 . The initial value of $t_{\max }$ set to 50 , and the parameter $a$ to compute $t_{\min }$ selected to $10^{5}$, while the maximum iteration of MMAS algorithm was set to 50 iterations.

With above setting for the parameters of algorithm, we run the algorithm five times in MATLAB environment, and the solution $J^{*}=0.13815$ obtained in average. The optimal control and state related to this solution are available in Figs. 1 and 2 respectively. The best known solution for this problem is $J^{*}=0.13317$ [12], and this solution can also be achieved when using MMAS method provided that the number of discretized points in either time or control intervals are large enough, as the number of these points increase the solution converge to the optimal solution.

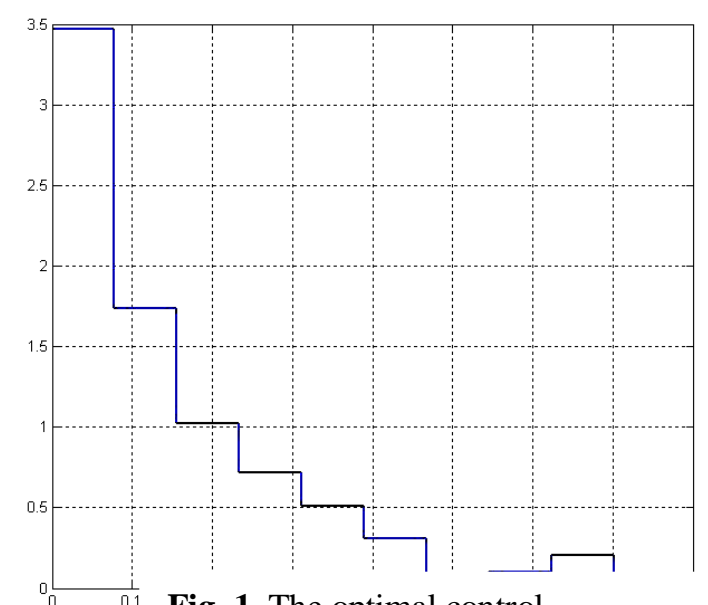

Fig. 1. The optimal control

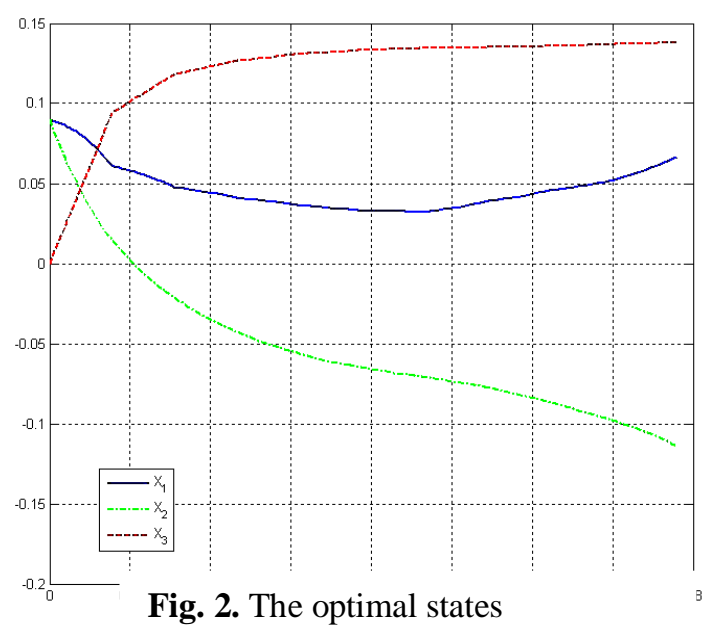

Fig. 2. The optimal states

\section{CONCLUSION}

This paper investigated to enhance the performance of MMAS algorithm with daemon actions to obtain approximate solution of optimal control problems. A discretization approach used to transfer the problem into a combinatorial parameter selection problem. Then, the Gaussian local search operator suggested as a daemon action in MMAS algorithm. The result of simulation shows the effectiveness of this method.

\section{Acknowledgements}

This study supported by the Malaysian Ministry of Higher Education and Research Management Centre of Universiti Teknologi Malaysia through research grant vot. no. 4B045. 


\section{REFERENCES}

[1] M., Birattari, G. D., Caro and M., Dorigo, Toward the formal foundation of Ant Programming. Proceedings of the International Workshop on Ant Algorithms (ANTS 2002). Third international workshop. Lecture Notes in Comput Sci, vol. 2463. Berlin, Springer; 2002.p. 188-201.

[2] M., Lopez-Ibanez, T. D., Prasad, and B., Paechter, Ant colony optimization for optimal control of pumps in water distribution networks. J. Water. Res. Pl-Asce. 134 (2008), no. 4, 337-346.

[3] A. H., Borzabadi and H. H., Mehne, Ant Colony Optimization for Optimal Control Problems. J. Inf. Comp. Sci. 4 (2009), no.4, 259264.

[4] V., Rishiwal, M., Yadav and K.V., Arya, Finding Optimal Paths on Terrain Maps using Ant Colony Algorithm. Int. J. Comp. Theory Eng. 2 (2010), no.3, 416-419.

[5] J. M.,van Ast, R.,Babuska,and B. De Schutter,Ant colony learning algorithm for optimal control. In Babuska, R. and Groen, F. C. A. (ed.) Interactive Collaborative Information Systems, volume 281 of Studies in Springer, Berlin / Heidelberg, pp. 155 - 182 , 2010 .

[6] M.,Dorigo and T.,Stutzle, Ant Colony Optimization. MIT Press, Cambridge, MA (2004).

[7] M.,Dorigo, V., Maniezzo and A., Colorni, Ant System: Optimization by a colony of cooperating agents, IEEE. T. Syst. Man. Cyb. PartB 26 (1996), no.1, 29-41.

[8] B., Bullnheimer,R. F., Hartl and C., Strauss, A new rank based version of the Ant System-A computational study. Technical report, Institute of Management Science University of Vienna, Austria, 1997.

[9] M., Dorigo and L. M., Gambardella, Ant Colony System: A cooperative learning approach to the traveling salesman problem. IEEE. T. Evolut. Comput. 1 (1997), no. 1, 53-66.

[10] T., Stützle and H. H., Hoos, The MAX-MIN Ant System and local search for the traveling salesman problem. In: Back, T., Michalewicz, Z., Yao, X. (eds.) Proceedings of the 1997 IEEE International Conference on Evolutionary Computation (ICEC'97), pp. 309-314. IEEE Press, Piscataway, NJ, 1997.

[11] T., Stützle and H. H., Hoos, MAX-MIN Ant System. Future. Gener. Comp. Sy. 16 (2000), no.8, 889-914.

[12] C. A., Floudas, et al., Handbook of Test Problems in Local and Global Optimization. Kluwer Academic Publishers. Dordrecht, (1999). 